\title{
Effect of Extra Contribution on Stochastic Optimal Investment Strategies for DC Pension with Stochastic Salary under the Affine Interest Rate Model
}

\author{
K. N. C. Njoku1, Bright O. Osu², Edikan E. Akpanibah ${ }^{3}$, Rosemary N. Ujumadu ${ }^{4}$ \\ ${ }^{1}$ Department of Mathematics, Imo State University, Owerri, Nigeria \\ ${ }^{2}$ Department of Mathematics, Michael Okpara University of Agriculture, Umudike, Nigeria \\ ${ }^{3}$ Department of Mathematics and Statistics, Federal University Otuoke, Yenagoa, Nigeria \\ ${ }^{4}$ Department of Mathematics, Chukwuemeka Odumegwu Ojukwu University, Uli, Nigeria \\ Email: osu.brjght@mouau.edu.ng,kevinnjoku60@yahoo.com, edikanakpanibah@gmail.com,rozyngujumadu@yahoo.com
}

How to cite this paper: Njoku, K.N.C., Osu, B.O., Akpanibah, E.E. and Ujumadu, R.N. (2017) Effect of Extra Contribution on Stochastic Optimal Investment Strategies for DC Pension with Stochastic Salary under the Affine Interest Rate Model. Journal of Mathematical Finance, 7, 821-833. https://doi.org/10.4236/jmf.2017.74043

Received: September 22, 2017

Accepted: October 22, 2017

Published: October 25, 2017

Copyright $\odot 2017$ by authors and Scientific Research Publishing Inc. This work is licensed under the Creative Commons Attribution International License (CC BY 4.0).

http://creativecommons.org/licenses/by/4.0/

\begin{abstract}
In this paper, optimal investment strategies for defined contribution (DC) Pension, with extra contribution are studied. Our model permits the plan member to make a defined extra contribution, as provided in the Nigerian Pension Reform Act of 2004. The plan member is free to invest in risk-free asset, and in two risky assets. A stochastic differential equation of the pension wealth that takes into account certain agreed proportions of the plan member's salary, paid as contribution, and extra contribution towards the pension fund, is presented. The Hamilton-Jacobi-Bellman (H-J-B) equation, Legend transformation, and Dual theory are used to obtain the explicit solution of the optimal investment strategies for constant relative risk aversion (CRRA) utility function. We observed that the plan member will increase the proportion of his wealth to be invested in bond and stock and will reduce the proportion to be invested in cash.
\end{abstract}

\section{Keywords}

Stochastic Optimal Control, Defined Contribution Pension,

Hamilton-Jacobi-Bellman Equation, Affine Interest Rate, Extra Contribution

\section{Introduction}

Recent publications in economic Journals and other reputable Mathematics and Science Journals have brought to light, different methods of optimizing invest- 
ment strategies and returns. Example, some researchers have made various contributions in this direction, particularly, in DC Pension Plan. [1] work on, stochastic life styling: optimal dynamic asset allocation for defined contribution pension plans. In their work, various properties and characteristics of the optimal asset allocation strategy, both with and without the presence of non-hedge able salary risk were discussed. The significance of alternative optimal strategy by pension providers was established.

In order to deal with optimal investment strategy, the need for maximization of the expected utility of the terminal wealth became necessary. Example, the Constant Relative Risk Aversion (CRRA) utility function, and (or) the Constant Absolute Risk Aversion (CARA) utility function were used to maximize the terminal wealth. [1]-[6] used CRRA to maximize terminal wealth. However, [6] used the CRRA and the CARA to maximize terminal wealth.

[7] applied the well-known H-J-B equation, Legend transform, and dual theory to obtain the explicit solutions of CRRA and CARA utility function, for the maximization of the terminal wealth. [8] took a different direction, where they considered an Inflationary market. In their work, the plan member made extra contribution to amortize the pension fund. The CRRA utility function was used to maximize the terminal wealth. This triggered our research. In our model; the amortization fund is a definite proportion of the plan member's salary.

The motive of this work is to maximize the optimal investment strategy for DC Pension with stochastic salary under the affine interest rate, with extra DC contribution, which is a proportion of the plan member's salary. We shall use the CRRA utility function to maximize our terminal wealth. Our approach is similar to that of Chubing [7], though, ours is an extension to extra DC contribution.

\section{Preliminaries}

We start with a complete and frictionless financial market that is continuously open over the fixed time interval $[0, T]$, for $T>0$ representing the retirement time of a given shareholder.

We assume that the market is made up of risk free asset (cash) a zero coupon bond and risky asset (stock). Let $(\Omega, F, P)$ be a complete probability space where $\Omega$ is a real space and $P$ is a probability measure, $\left\{W_{r}(t), W_{s}(t): t \geq 0\right\}$ is a standard two dimensional Brownian motion such that they are orthogonal to each other. $F$ is the filtration and denotes the information structure generated by the Brownian motions $\left\{W_{r}(t), W_{s}(t)\right\}$.

Let $C(t)$ denote the price of the risk free asset at time $t$ and it is modeled as follows

$$
\frac{\mathrm{d} C(t)}{C(t)}=r(t) \mathrm{d} t, \quad C(0)=1
$$

$r(t)$ is the short interest rate process and is given by the stochastic differen- 
tial equation $(\mathrm{SDE})$

$$
\begin{gathered}
\mathrm{d} r(t)=(a-b r(t)) \mathrm{d} t-\sigma_{r} \mathrm{~d} W_{r}(t), \\
\sigma_{r}=\sqrt{c_{1} r(t)+c_{2}}, t \geq 0,
\end{gathered}
$$

where $a, b, r(0), c_{1}$ and $c_{2}$ are positive real numbers. If $c_{1}$ (resp., $c_{2}$ ) is equal to zero, we have a special case, as in [9] [10]. So under these dynamics, the term structure of the short interest rates is affine, which has been studied by [2] [7] [11] [12].

Let $S(t)$ denote the price of the risky asset and its dynamics is given based on a continuous time stochastic process at $t \geq 0$ and the dynamics of the price process is described as follows

$$
\frac{\mathrm{d} S(t)}{S(t)}=r(t) \mathrm{d} t+\sigma_{s}\left(\mathrm{~d} W_{s}(t)+\lambda_{1} \mathrm{~d} t\right)+n_{1} \sigma_{r}\left(\mathrm{~d} W_{r}(t)+\lambda_{2} \sigma_{r} \mathrm{~d} t\right), S(0)=S_{0}
$$

with $\lambda_{1}, \lambda_{2}$ (resp., $\sigma_{s}, n_{1}$ ) being constants (resp., positive constants) see [2] [4] [7].

A zero-coupon bond with maturity $T$, whose price at time $t$ is denoted by $B(t, T), t \geq 0$, and

Its dynamics is given by the SDE below see [2] [4]

$$
\frac{\mathrm{d} B(t, T)}{B(t, T)}=r(t) \mathrm{d} t+\sigma_{B}(T-t, r(t))\left(\mathrm{d} W_{r}(t)+\lambda_{2} \sigma_{r} \mathrm{~d} t\right), B(T, T)=1
$$

where $\sigma_{B}(T-t, r(t))=f(T-t) \sigma_{r}$ and

$$
f(t)=\frac{2\left(\mathrm{e}^{m t}-1\right)}{m-\left(b-c_{1} \lambda_{2}\right)+\mathrm{e}^{m t}\left(m+b-c_{1} \lambda_{2}\right)}, m=\sqrt{\left(b-c_{1} \lambda_{2}\right)^{2}+2 c_{1}}
$$

Based on the works of [1] [4] [7] [12] we denote the stochastic salary at time $t$ by $(t)$ which is described by

$$
\frac{\mathrm{d} L(t)}{L(t)}=\mu_{L}(t, r(t)) \mathrm{d} t+n_{2} \sigma_{r} \mathrm{~d} W_{r}(t)+n_{3} \sigma_{s} \mathrm{~d} W_{s}(t), L(0)=L_{0}
$$

where $n_{2}, n_{3}$ are real constants, which are two volatility scale factors measuring how the risk sources of interest rate and stock affect the salary. That is to say, the salary volatility is supposed to be a hedge able volatility whose risk source belongs to the set of the financial market risk sources. This is in accordance with the assumption [4], but is differs from those of [1] [12] who also suggest that the salary was influence by non hedgeable risk source (i.e., non-financial market). Also [7] assume that the instantaneous mean of the salary is such that $\mu_{L}(t, r(t))=r(t)+m_{L}$ where $m_{L}$ is a real constant.

\section{Methodology}

\subsection{Hamilton-Jacobi-Bellman (HJB) Equation}

Suppose, we represent $u=\left(u_{B}, u_{S}\right)$ as the strategy and we define the utility attained by the contributor from aiven state $x$ at time $t$ as 


$$
G_{u}(t, r, x)=E_{u}[V(X(T)) \mid r(t)=r, X(t)=x],
$$

where $t$ is the time, $r$ is the short interest rate and $x$ is the wealth. Our interest here is to find the optimal value function

$$
G(t, r, x)=\sup _{u} G_{u}(t, r, x)
$$

and the optimal strategy $u^{*}=\left(u_{B}^{*}, u_{S}^{*}\right)$ such that

$$
G_{u^{*}}(t, r, x)=G(t, r, x) \text {. }
$$

\subsection{Legendre Transformation}

The Legendre transform and dual theory help to transform non linear partial differential equation into a linear partial differential equation.

Theorem 3.1: Let $f: R^{n} \rightarrow R$ be a convex function for $z>0$, define the Legendre transform

$$
L(z)=\max _{x}\{f(x)-z x\}
$$

where $L(z)$ is the Legendre dual of $f(x)$ [13].

Since $f(x)$ is convex, from theorem 3.1 we defined the Legendre transform

$$
\hat{G}(t, r, z)=\sup _{x>0}\{G(t, r, x)-z x \mid 0<x<\infty\}, 0<t<T .
$$

where $\hat{G}$ is the dual of $G$ and $z>0$ is the dual variable of $x$.

The value of $x$ where this optimum is attained is denoted by $h(t, r, z)$, so that

$$
h(t, r, z)=\inf _{x>0}\{x \mid G(t, r, x) \geq z x+\hat{G}(t, r, z)\}, 0<t<T .
$$

The function $h$ and $\hat{G}$ are closely related and can be refers to either one of them as the dual of $G$. These functions are related as follows

$$
\hat{G}(t, r, z)=G(t, r, h)-z h
$$

where

$$
h(t, r, z)=x, G_{x}=z \text {, and relating } \hat{G} \text { to } h \text { by } h=-\hat{G}_{z}
$$

At terminal time, we denote

$$
\hat{V}(z)=\sup _{w>0}\{V(w)-z w \mid 0<w<\infty\},
$$

and

$$
H(z)=\sup _{w>0}\{w \mid V(w) \geq z w+\hat{V}(z)\} .
$$

As a result

$$
H(z)=\left(V^{\prime}\right)^{-1}(z),
$$

where $H$ is the inverse of the marginal utility $V$ and note that $G(T, r, x)=V(x)$

At terminal time $T$, we can define

$$
\begin{aligned}
& h(T, r, z)=\inf _{x>0}\{x \mid V(x) \geq z x+\hat{G}(T, r, z)\} \\
& \text { and } \hat{G}(T, r, z)=\sup _{x>0}\{V(x)-z x\}
\end{aligned}
$$


so that

$$
h(T, r, z)=\left(V^{\prime}\right)^{-1}(z)
$$

\section{Model Formulation}

Here the contributions are continuously paid into the pension fund at the rate of $\left(e_{1}+e_{2}\right) L(t)$ where $e_{1}$ is the mandatory rate of contribution and $e_{2}$ is the extra contribution rate which is assumed to be at constant rate. Let $W(t)$ denote the wealth of pension fund at time $t \in[0, T] . u_{B}(t)$ and $u_{S}(t)$ represent the proportion of the pension fund invested in the bond and the stock respectively. This implies that the proportion of the pension fund invested in the risk-free asset $u_{C}(t)=1-u_{B}(t)-u_{S}(t)$. The dynamics of the pension wealth is given by

$$
\begin{aligned}
\mathrm{d} W(t)= & u_{C} W(t) \frac{\mathrm{d} C(t)}{C(t)}+u_{B} W(t) \frac{\mathrm{d} B(t, T)}{B(t, T)}+u_{S} W(t) \frac{\mathrm{d} S(t)}{S(t)} \\
& +\left(e_{1}+e_{2}\right) L(t) \mathrm{d} t ; e_{2} \in[0,1)
\end{aligned}
$$

Substituting (1), (3) and (4) in (17) we have

$$
\begin{aligned}
\mathrm{d} W(t)= & W(t)\left[r(t)+\lambda_{2} \sigma_{r} \sigma_{B} u_{B}+u_{S}\left(\lambda_{1} \sigma_{S}+\lambda_{2} \sigma_{r}^{2} n_{1}\right)\right] \mathrm{d} t\left(e_{1}+e_{2}\right) L(t) \mathrm{d} t \\
& +W(t)\left(\sigma_{B} u_{B}+u_{S} \sigma_{r} n_{1}\right) \mathrm{d} W_{r}(t)+W(t) \sigma_{S} u_{S} \mathrm{~d} W_{S}(t)
\end{aligned}
$$

Let the relative wealth be defined as follows

$$
X(t)=\frac{W(t)}{L(t)}
$$

Applying product rule and Ito's formula to (19) and making use of (6) and (18) we arrive at the following equation

$$
\begin{gathered}
\mathrm{d} X(t)=X(t)\left[r(t)-\mu_{L}+n_{2}^{2} \sigma_{r}^{2}+n_{3}^{2} \sigma_{S}^{2}+\sigma_{B} u_{B} \sigma_{r}\left(\lambda_{2}-n_{2}\right)\right. \\
\left.+u_{S}\left(\lambda_{1} \sigma_{s}+\lambda_{2} \sigma_{r}^{2} n_{1}+\sigma_{S}^{2} n_{3}-n_{1} n_{2} \sigma_{r}^{2}\right)\right] \mathrm{d} t+\left(e_{1}+e_{2}\right) \mathrm{d} t \\
+X(t)\left(\sigma_{B} u_{B}+n_{1} \sigma_{r} u_{S}-n_{2} \sigma_{r}\right) \mathrm{d} W_{r}(t) \\
+\left(X(t)\left(u_{S}-n_{3}\right) \sigma_{s}\right) \mathrm{d} W_{S}(t), X(0)=W(0) / L(0) \\
\mathrm{d} X(t)=X\left(\theta_{1}+\theta_{2} u_{B}+\theta_{3} u_{S}\right) \mathrm{d} t+\left(e_{1}+e_{2}\right) \mathrm{d} t \\
+X\left(\sigma_{B} u_{B}+n_{1} \sigma_{r} u_{S}-n_{2} \sigma_{r}\right) \mathrm{d} W_{r}(t)+\left(X\left(u_{S}-n_{3}\right) \sigma_{s}\right) \mathrm{d} W_{S}(t) \\
\theta_{1}=r(t)-\mu_{L}+n_{2}^{2} \sigma_{r}^{2}+n_{3}^{2} \sigma_{S}^{2} \\
\theta_{2}=\sigma_{B} \sigma_{r}\left(\lambda_{2}-n_{2}\right) \\
\theta_{3}=\lambda_{1} \sigma_{s}+\lambda_{2} \sigma_{r}^{2} n_{1}+\sigma_{S}^{2} n_{3}-n_{1} n_{2} \sigma_{r}^{2}
\end{gathered}
$$

The Hamilton-Jacobi-Bellman (HJB) equation associated with (21) is

$$
\begin{aligned}
& G_{t}+(a-b r) G_{r}+\frac{1}{2} \sigma_{r}^{2} G_{r r}+\sup _{u}\left\{\left[\left(x\left(\theta_{1}+u_{B} \theta_{2}+u_{S} \theta_{3}\right)\right)+\left(e_{1}+e_{2}\right)\right] G_{x}\right. \\
& +\frac{1}{2}\left(x\left(u_{S}-n_{3}\right) \sigma_{s}\right)^{2} G_{x x}+\frac{1}{2}\left(x\left(\sigma_{B} u_{B}+n_{1} \sigma_{r} u_{S}-n_{2} \sigma_{r}\right)\right)^{2} G_{x x} \\
& \left.-x \sigma_{r}\left(\sigma_{B} u_{B}+n_{1} \sigma_{r} u_{S}-n_{2} \sigma_{r}\right) G_{r x}\right\}=0
\end{aligned}
$$


where $G_{t}, G_{r}, G_{x}, G_{r x}, G_{r x}$ and $G_{x x}$ are partial derivatives of first and second orders with respect to time, short interest rate, and relative wealth.

Differentiating (23) with respect to $u_{B}$ and $u_{S}$, we obtain the first-order maximizing conditions for the optimal strategies $u_{B}^{*}$ and $u_{S}^{*}$ as

$$
\begin{gathered}
\theta_{2} G_{x}+x \sigma_{B}\left(\sigma_{B} u_{B}^{*}+n_{1} \sigma_{r} u_{S}^{*}-n_{2} \sigma_{r}\right) G_{x x}-\sigma_{r} \sigma_{B} G_{r x}=0 \\
\theta_{3} G_{x}+x n_{1} \sigma_{r}\left(\sigma_{B} u_{B}^{*}+n_{1} \sigma_{r} u_{S}^{*}-n_{2} \sigma_{r}\right) G_{x x}+x\left(u_{S}^{*}-n_{3}\right) \sigma_{S} G_{x x}-n_{1} \sigma_{r}^{2} G_{r x}=0
\end{gathered}
$$

Solving (24) and (25) simultaneously we have

$$
\begin{gathered}
u_{S}^{*}=n_{3}-\left(\frac{\lambda_{1}+n_{3} \sigma_{S}^{2}}{x \sigma_{S}}\right) \frac{G_{x}}{G_{x x}} \\
u_{B}^{*}=\frac{\sigma_{r}\left(n_{2}-n_{1} n_{3}\right)}{\sigma_{B}}+\left(\frac{\theta_{4} \sigma_{r}}{x \sigma_{B}}\right) \frac{G_{x}}{G_{x x}}+\left(\frac{\sigma_{r}}{x \sigma_{B}}\right) \frac{G_{r x}}{G_{x x}} \\
\theta_{4}=\frac{\sigma_{S} n_{2}+n_{1} \lambda_{1}+n_{1} n_{3} \sigma_{S}^{2}-\lambda_{2} \sigma_{S}}{\sigma_{S}}
\end{gathered}
$$

Substituting (26) and (27) into (23) we have

$$
\begin{gathered}
G_{t}+(a-b r) G_{r}+\frac{1}{2} \sigma_{r}^{2} G_{r r}+\left(\rho_{0}+\rho_{1} x\right) G_{x} \\
+\left(\rho_{2}-\frac{1}{2}\left(\lambda_{2}-n_{2}\right)^{2} \sigma_{r}^{2}\right) \frac{G_{x}^{2}}{G_{x x}}+\left(\lambda_{2}-n_{2}\right) \sigma_{r}^{2} \frac{G_{x} G_{r x}}{G_{x x}}-\frac{1}{2} \sigma_{r}^{2} \frac{G_{r x}}{G_{x x}}=0 \\
\rho_{0}=e_{1}+e_{2} \\
\rho_{1}=n_{3} \lambda_{1} \sigma_{s}+\lambda_{2} \sigma_{r}^{2} n_{2}+2 \sigma_{S}^{2} n_{3}^{2}-m_{L} \\
\rho_{2}=\frac{1}{2} \sigma_{S}^{4} n_{3}^{2}-\sigma_{S}^{3} n_{3}^{2}-n_{3} \sigma_{s} \lambda_{1}-\frac{1}{2} \lambda_{1}^{2}
\end{gathered}
$$

Applying Legendre transform to (28) we have

$$
\begin{gathered}
\hat{G}_{t}+(a-b r) \hat{G}_{r}+\frac{1}{2} \sigma_{r}^{2} \hat{G}_{r r}+\left(\rho_{0}+\rho_{1} x\right) z \\
-\left(\rho_{2}-\frac{1}{2}\left(\lambda_{2}-n_{2}\right)^{2} \sigma_{r}^{2}\right) z^{2} \hat{G}_{z z}+\left(\lambda_{2}-n_{2}\right) \sigma_{r}^{2} z \hat{G}_{r z}=0 \\
\rho_{0}=e_{1}+e_{2} \\
\rho_{1}=n_{3} \lambda_{1} \sigma_{s}+\lambda_{2} \sigma_{r}^{2} n_{2}+2 \sigma_{S}^{2} n_{3}^{2}-m_{L} \\
\rho_{2}=\frac{1}{2} \sigma_{S}^{4} n_{3}^{2}-\sigma_{S}^{3} n_{3}^{2}-n_{3} \sigma_{s} \lambda_{1}-\frac{1}{2} \lambda_{1}^{2}
\end{gathered}
$$

Differentiating Equation (30) for $\hat{G}$ with respect to $z$ we obtain a linear PDE in terms of $h$ and its derivatives and using $x=h=\hat{G}_{z}$, we have

$$
\begin{aligned}
& h_{t}+(a-b r) h_{r}+\frac{1}{2} \sigma_{r}^{2} h_{r r}-\rho_{0}-\rho_{1} h-\rho_{1} z h_{z}+\left(\lambda_{2}-n_{2}\right) \sigma_{r}^{2} h_{r} \\
& +\left(\lambda_{2}-n_{2}\right) \sigma_{r}^{2} z h_{r z}-2\left(\rho_{2}-\frac{1}{2}\left(\lambda_{2}-n_{2}\right)^{2} \sigma_{r}^{2}\right) z h_{z} \\
& -\left(\rho_{2}-\frac{1}{2}\left(\lambda_{2}-n_{2}\right)^{2} \sigma_{r}^{2}\right) z^{2} h_{z z}=0
\end{aligned}
$$


where

$$
\begin{gathered}
\rho_{0}=e_{1}+e_{2} \\
\rho_{1}=n_{3} \lambda_{1} \sigma_{s}+\lambda_{2} \sigma_{r}^{2} n_{2}+2 \sigma_{S}^{3} n_{3}^{2}-m_{L} \\
\rho_{2}=\frac{1}{2} \sigma_{S}^{4} n_{3}^{2}-\sigma_{S}^{3} n_{3}^{2}-n_{3} \sigma_{s} \lambda_{1}-\frac{1}{2} \lambda_{1}^{2} \\
u_{C}=1-u_{B}-u_{S} \\
u_{S}^{*}=n_{3}-\left(\frac{\lambda_{1}+n_{3} \sigma_{S}^{2}}{h \sigma_{S}}\right) z h_{z} \\
u_{B}^{*}=\frac{n_{2}-n_{1} n_{3}}{f(T-t)}+\left(\frac{\theta_{4}}{h f(T-t)}\right) z h_{z}-\left(\frac{h_{r}}{h f(T-t)}\right) \\
\theta_{4}=\frac{\sigma_{S} n_{2}+n_{1} \lambda_{1}+n_{1} n_{3} \sigma_{S}^{2}-\lambda_{2} \sigma_{S}}{\sigma_{S}} \\
2(t)=\frac{2\left(\mathrm{e}^{m t}-1\right)}{m-\left(b-c_{1} \lambda_{2}\right)+\mathrm{e}^{m t}\left(m+b-c_{1} \lambda_{2}\right)}, m=\sqrt{\left(b-c_{1} \lambda_{2}\right)^{2}+2} c_{1}
\end{gathered}
$$

We will now solve (31) for $h$ and substitute into (33) and (34) to obtain the optimal investment strategies.

\section{Explicit Solution of the Optimal Investment Strategies for the CRRA Utility Function}

Assume the investor takes a power utility function

$$
V(x)=\frac{x^{p}}{p}, p<1, p \neq 0
$$

The relative risk aversion of an investor with utility described in (37) is constant and (37) is a CRRA utility.

From (16) we have $h(T, r, z)=\left(V^{\prime}\right)^{-1}(z)$ and from (37) we have

$$
h(T, r, z)=z^{\frac{1}{p-1}}
$$

We assume a solution to (31) with the following form

$$
h(t, r, z)=v(t, r)\left[z^{\frac{1}{p-1}}\right]+y(t), y(T)=0, v(T, s)=1 .
$$

Then

$$
\begin{gathered}
h_{t}=v_{t} z^{\frac{1}{p-1}}+y^{\prime}, h_{z}=-\frac{v}{1-p} z^{\left(\frac{1}{p-1}-1\right)}, h_{r z}=-\frac{v_{r}}{1-p} z^{\left(\frac{1}{p-1}-1\right)}, \\
h_{z z}=\frac{(2-p) v}{(1-p)^{2}} z^{\left(\frac{1}{p-1}-1\right)}, h_{r}=v_{r} z^{\frac{1}{p-1}}, h_{r r}=v_{r r} z^{\frac{1}{p-1}} .
\end{gathered}
$$

Substituting (39) into (31) we have 


$$
\begin{aligned}
& \left\{v_{t}+(a-b r) v_{r}-\frac{\left(\lambda_{2}-n_{2}\right) p \sigma_{r}^{2}}{1-p} v_{r}+\frac{1}{2} \sigma_{r}^{2} v_{r r}+\frac{\rho_{1} p v}{1-p}\right. \\
& \left.-\frac{p v}{(1-p)^{2}}\left(\rho_{2}-\frac{1}{2}\left(\lambda_{2}-n_{2}\right)^{2} \sigma_{r}^{2}\right)\right\} z^{\frac{1}{p-1}}+y^{I}(t)-\rho_{1} y(t)-\rho_{0}=0
\end{aligned}
$$

Splitting (40) we have

$$
\begin{gathered}
y^{I}(t)-\rho_{1} y(t)-\rho_{0}=0 \\
v_{t}+(a-b r) v_{r}-\frac{\left(\lambda_{2}-n_{2}\right) p \sigma_{r}^{2}}{1-p} v_{r}+\frac{1}{2} \sigma_{r}^{2} v_{r r}+\frac{\rho_{1} p v}{1-p} \\
-\frac{p v}{(1-p)^{2}}\left(\rho_{2}-\frac{1}{2}\left(\lambda_{2}-n_{2}\right)^{2} \sigma_{r}^{2}\right)=0
\end{gathered}
$$

Solving (41) with the given boundary condition $y(T)=0$

$$
y(t)=-\frac{\rho_{0}}{\rho_{1}}\left(1-\mathrm{e}^{-\rho_{1}(T-t)}\right)
$$

To solve (42), we conjecture a solution of the form

$$
\begin{gathered}
v(t, r)=M(t) \mathrm{e}^{N(t) r}, M(T)=1, N(T)=0 \\
v_{r}=M(t) N(t) \mathrm{e}^{N(t) r}, v_{r r}=M(t) N^{2}(t) \mathrm{e}^{N(t) r} \\
\text { and } v_{t}=r M(t) N^{I}(t) \mathrm{e}^{N(t) r}+M^{I}(t) \mathrm{e}^{N(t) r}
\end{gathered}
$$

Substituting (44) into (42), we have

$$
\begin{aligned}
& \frac{M^{I}}{M}+\frac{a-\left(\left(\lambda_{2}-n_{2}\right) c_{1}+a\right) p}{1-p} N+\frac{1}{2} c_{2} N^{2} \\
& +\frac{p\left(\rho_{1}-\rho_{2}-p \rho_{1}\right)}{(1-p)^{2}}+\frac{1}{2} \frac{\left(\lambda_{2}-n_{2}\right)^{2} p c_{2}}{(1-p)^{2}} \\
& +r\left(N^{I}-\frac{b+\left(\left(\lambda_{2}-n_{2}\right) c_{1}-b\right) p}{1-p} N+\frac{1}{2} c_{1} N^{2}+\frac{1}{2} \frac{\left(\lambda_{2}-n_{2}\right)^{2} p c_{1}}{(1-p)^{2}}\right)
\end{aligned}
$$

Splitting (44) we have

$$
\begin{gathered}
\frac{M^{I}}{M}+\frac{a-\left(\left(\lambda_{2}-n_{2}\right) c_{1}+a\right) p}{1-p} N+\frac{1}{2} c_{2} N^{2} \\
+\frac{p\left(\rho_{1}-\rho_{2}-p \rho_{1}\right)}{(1-p)^{2}}+\frac{1}{2} \frac{\left(\lambda_{2}-n_{2}\right)^{2} p c_{2}}{(1-p)^{2}}=0 \\
N^{I}-\frac{b+\left(\left(\lambda_{2}-n_{2}\right) c_{1}-b\right) p}{1-p} N+\frac{1}{2} c_{1} N^{2}+\frac{1}{2} \frac{\left(\lambda_{2}-n_{2}\right)^{2} p c_{1}}{(1-p)^{2}}=0 \\
N(t)=\frac{d_{1}-d_{2} \mathrm{e}^{\frac{1}{2} c_{1}\left(d_{1}-d_{2}\right)(T-t)}}{1-\frac{d_{1}}{d_{2}} \mathrm{e}^{\frac{1}{2} c_{1}\left(d_{1}-d_{2}\right)(T-t)}}
\end{gathered}
$$

where 


$$
\begin{aligned}
& \left.M(t)=\mathrm{e}^{\left\{\frac{\left(\left(\lambda_{2}-n_{2}\right) c_{1}+a\right) p-a}{1-p} \int N(t) \mathrm{d} t-\frac{1}{2} c_{2} \int N^{2}(t) \mathrm{d} t-\frac{p\left(\rho_{1}-\rho_{2}-p \rho_{1}\right)}{(1-p)^{2}} t+C\right.}\right\}, M(T)=1 \\
& d_{1}=\frac{\left(b+\left(\left(\lambda_{2}-n_{2}\right) c_{1}-b\right) p+\sqrt{\left(b+\left(\left(\lambda_{2}-n_{2}\right) c_{1}-b\right) p\right)^{2}-\left(\lambda_{2}-n_{2}\right)^{2} p c_{1}^{2}}\right)}{(1-p) c_{1}} \\
& d_{2}=\frac{\left(b+\left(\left(\lambda_{2}-n_{2}\right) c_{1}-b\right) p-\sqrt{\left(b+\left(\left(\lambda_{2}-n_{2}\right) c_{1}-b\right) p\right)^{2}-\left(\lambda_{2}-n_{2}\right)^{2} p c_{1}^{2}}\right)}{(1-p) c_{1}} \\
& v(r, t)=\exp \left\{\left(\frac{\left(\left(\lambda_{2}-n_{2}\right) c_{1}+a\right) p-a}{1-p} \int \frac{d_{1}-d_{2} \mathrm{e}^{\frac{1}{2} c_{1}\left(d_{1}-d_{2}\right)(T-t)}}{1-\frac{d_{1}}{d_{2}} \mathrm{e}^{\frac{1}{2} c_{1}\left(d_{1}-d_{2}\right)(T-t)}}\right) \mathrm{d} t\right. \\
& \left.-\frac{1}{2} c_{2} \int\left(\frac{d_{1}-d_{2} \mathrm{e}^{\frac{1}{2} c_{1}\left(d_{1}-d_{2}\right)(T-t)}}{1-\frac{d_{1}}{d_{2}} \mathrm{e}^{\frac{1}{2} c_{1}\left(d_{1}-d_{2}\right)(T-t)}}\right)^{2} \mathrm{~d} t-\frac{p\left(\rho_{1}-\rho_{2}-p \rho_{1}\right)}{(1-p)^{2}} t+C\right) \\
& \left.+\left(\frac{d_{1}-d_{2} \mathrm{e}^{\frac{1}{2} c_{1}\left(d_{1}-d_{2}\right)(T-t)}}{1-\frac{d_{1}}{d_{2}} \mathrm{e}^{\frac{1}{2} c_{1}\left(d_{1}-d_{2}\right)(T-t)}}\right) r\right\}
\end{aligned}
$$

Hence the solution of (31) is given as

$$
\begin{aligned}
h(t, r, z)= & \exp \left\{\left(\frac{\left(\left(\lambda_{2}-n_{2}\right) c_{1}+a\right) p-a}{1-p} \int\left(\frac{d_{1}-d_{2} \mathrm{e}^{\frac{1}{2} c_{1}\left(d_{1}-d_{2}\right)(T-t)}}{1-\frac{d_{1}}{d_{2}} \mathrm{e}^{\frac{1}{2} c_{1}\left(d_{1}-d_{2}\right)(T-t)}}\right) \mathrm{d} t\right.\right. \\
& \left.-\frac{1}{2} c_{2} \int\left(\frac{d_{1}-d_{2} \mathrm{e}^{\frac{1}{2} c_{1}\left(d_{1}-d_{2}\right)(T-t)}}{1-\frac{d_{1}}{d_{2}} \mathrm{e}^{\frac{1}{2} c_{1}\left(d_{1}-d_{2}\right)(T-t)}}\right) \mathrm{d} t-\frac{p\left(\rho_{1}-\rho_{2}-p \rho_{1}\right)}{(1-p)^{2}} t+C\right) \\
& \left.\left.+\left(\frac{d_{1}-d_{2} \mathrm{e}^{\frac{1}{2} c_{1}\left(d_{1}-d_{2}\right)(T-t)}}{1-\frac{d_{1}}{d_{2}} \mathrm{e}^{\frac{1}{2} c_{1}\left(d_{1}-d_{2}\right)(T-t)}}\right) r\right\} z^{\frac{1}{p-1}}\right]-\frac{\rho_{0}}{\rho_{1}}\left(1-\mathrm{e}^{-\rho_{1}(T-t)}\right)
\end{aligned}
$$

\section{Proposition 5.1}

The optimal investment strategies for cash, bond and stock is given as follows

$$
\begin{gathered}
u_{C}^{*}=1-u_{B}^{*}-u_{S}^{*} \\
u_{S}^{*}=n_{3}+\left(\frac{\lambda_{1}+n_{3} \sigma_{S}^{2}}{(1-p) \sigma_{S}}\right)+\left(\frac{\lambda_{1}+n_{3} \sigma_{S}^{2}}{(1-p) \sigma_{S}}\right)\left[\frac{\left(e_{1}+e_{2}\right) l}{w}\left(\frac{1-\mathrm{e}^{-\rho_{1}(T-t)}}{\rho_{1}}\right)\right]
\end{gathered}
$$




$$
\begin{gathered}
u_{B}^{*}=\frac{1}{f(T-t)}\left\{\left(n_{2}-n_{1} n_{3}\right)-\frac{1}{1-p}\left(\frac{w \rho_{1}+\left(e_{1}+e_{2}\right) l}{w \rho_{1}}\right)\left(\theta_{4}+(1-p) N(t)\right)\left(\left(1-\mathrm{e}^{-\rho_{1}(T-t)}\right)\right\}\right. \\
\theta_{4}=\frac{\sigma_{S} n_{2}+n_{1} \lambda_{1}+n_{1} n_{3} \sigma_{S}^{2}-\lambda_{2} \sigma_{S}}{\sigma_{S}} \\
\rho_{0}=e_{1}+e_{2} \\
\rho_{1}=n_{3} \lambda_{1} \sigma_{s}+\lambda_{2} \sigma_{r}^{2} n_{2}+2 \sigma_{S}^{2} n_{3}^{2}-m_{L} \\
\rho_{2}=\frac{1}{2} \sigma_{S}^{4} n_{3}^{2}-\sigma_{S}^{3} n_{3}^{2}-n_{3} \sigma_{s} \lambda_{1}-\frac{1}{2} \lambda_{1}^{2} \\
f(t)=\frac{2\left(\mathrm{e}^{m t}-1\right)}{m-\left(b-c_{1} \lambda_{2}\right)+\mathrm{e}^{m t}\left(m+b-c_{1} \lambda_{2}\right)}, m=\sqrt{\left(b-c_{1} \lambda_{2}\right)^{2}+2} c_{1} \\
N(t)=\frac{d_{1}-d_{2} \mathrm{e}^{\frac{1}{2} c_{1}\left(d_{1}-d_{2}\right)(T-t)}}{1-\frac{d_{1}}{d_{2}} \mathrm{e}^{\frac{1}{2} c_{1}\left(d_{1}-d_{2}\right)(T-t)}} \\
d_{1}=\frac{\left(b+\left(\left(\lambda_{2}-n_{2}\right) c_{1}-b\right) p+\sqrt{\left(b+\left(\left(\lambda_{2}-n_{2}\right) c_{1}-b\right) p\right)^{2}-\left(\lambda_{2}-n_{2}\right)^{2} p c_{1}^{2}}\right)}{(1-p) c_{1}} \\
d_{2}=\frac{\left(b+\left(\left(\lambda_{2}-n_{2}\right) c_{1}-b\right) p-\sqrt{\left(b+\left(\left(\lambda_{2}-n_{2}\right) c_{1}-b\right) p\right)^{2}-\left(\lambda_{2}-n_{2}\right)^{2} p c_{1}^{2}}\right)}{(1-p) c_{1}}
\end{gathered}
$$

\section{Remark 5.1}

Suppose $n_{2}=n_{3}=0, p \rightarrow 0, l=1$, then the salary is not stochastic and the optimal investment is given as

$$
\begin{gathered}
u_{S}^{*}=\left(\frac{\lambda_{1}}{\sigma_{S}}\right)+\left(\frac{\lambda_{1}}{\sigma_{S}}\right)\left[\frac{e_{1}+e_{2}}{w}\left(\frac{1-\mathrm{e}^{-\rho_{1}(T-t)}}{\rho_{1}}\right)\right] \\
u_{B}^{*}=\frac{1}{f(T-t)}\left\{\left(\frac{\lambda_{2} \sigma_{S}-n_{1} \lambda_{1}}{\sigma_{S}}\right)\left(\frac{w \rho_{1}+\left(e_{1}+e_{2}\right)}{w \rho_{1}}\right)\left(1-\mathrm{e}^{-\rho_{1}(T-t)}\right)\right\}
\end{gathered}
$$

Furthermore, assume there is no extra contribution i.e., $e_{2}=0$ we have

$$
\begin{gathered}
u_{S_{1}}^{*}=\left(\frac{\lambda_{1}}{\sigma_{S}}\right)+\left(\frac{\lambda_{1}}{\sigma_{S}}\right)\left[\frac{e_{1}}{w}\left(\frac{1-\mathrm{e}^{-\rho_{1}(T-t)}}{\rho_{1}}\right)\right] \\
u_{B_{1}}^{*}=\frac{1}{f(T-t)}\left\{\left(\frac{\lambda_{2} \sigma_{S}-n_{1} \lambda_{1}}{\sigma_{S}}\right)\left(\frac{w \rho_{1}+e_{1}}{w \rho_{1}}\right)\left(1-\mathrm{e}^{-\rho_{1}(T-t)}\right)\right\}
\end{gathered}
$$

\section{Proposition 5.2}

Suppose $u_{S_{1}}^{*}<u_{S}^{*}$ and $u_{B_{1}}^{*}<u_{B}^{*}$ then $u_{C_{1}}^{*}>u_{C}^{*}$

Proof

Since $u_{S_{1}}^{*}<u_{S}^{*}$ and $u_{B_{1}}^{*}<u_{B}^{*}$ then,

$$
u_{S_{1}}^{*}+u_{B_{1}}^{*}<u_{S}^{*}+u_{B}^{*}
$$




$$
\begin{gathered}
1-\left(u_{S_{1}}^{*}+u_{B_{1}}^{*}\right)>1-\left(u_{S}^{*}+u_{B}^{*}\right) \\
1-u_{S_{1}}^{*}-u_{B_{1}}^{*}>1-u_{S}^{*}-u_{B}^{*}
\end{gathered}
$$

But $u_{C_{1}}^{*}=1-u_{S_{1}}^{*}-u_{B_{1}}^{*}$ and $u_{C}^{*}=1-u_{S}^{*}-u_{B}^{*}$, therefore

$$
u_{C_{1}}^{*}>u_{C}^{*}
$$

\section{Remark 5.2}

Suppose $e_{2}=0$ then the strategies is without the extra contribution hence the required strategies are as given below

$$
\begin{gathered}
u_{S_{2}}^{*}=n_{3}+\left(\frac{\lambda_{1}+n_{3} \sigma_{S}^{2}}{(1-p) \sigma_{S}}\right)+\left(\frac{\lambda_{1}+n_{3} \sigma_{S}^{2}}{(1-p) \sigma_{S}}\right)\left[\frac{\left(e_{1}\right) l}{w}\left(\frac{1-\mathrm{e}^{-\rho_{1}(T-t)}}{\rho_{1}}\right)\right] \\
u_{S_{2}}^{*}=\frac{1}{f(T-t)}\left\{\left(n_{2}-n_{1} n_{3}\right)-\frac{1}{1-p}\left(\frac{w \rho_{1}+\left(e_{1}\right) l}{w \rho_{1}}\right)\left(\theta_{4}+(1-p) N(t)\right)\left(1-\mathrm{e}^{-\rho_{1}(T-t)}\right)\right\}
\end{gathered}
$$

The result above is similar to that in [7].

Case 1

If $n_{3}>0, \lambda_{1}>0, \sigma_{S}>0,0<p<1, \rho_{1}>0, l>0, e_{2}=0$ then $u_{S_{2}}^{*}<u_{S}^{*}$

Case 2

If $\left(n_{2}-n_{1} n_{3}\right)>0, f(T-t)>0, \lambda_{1}>0, \sigma_{s}>0,0<p<1, \rho_{1}>0, l>0, N(t)>0$, $e_{2}=0$

then $u_{B_{2}}^{*}<u_{B}^{*}$

Case 2

If $\left(n_{2}-n_{1} n_{3}\right)<0, f(T-t)>0, \lambda_{1}>0, \sigma_{S}>0,0<p<1, \rho_{1}>0, l>0, N(t)>0$, $e_{2}=0$

then $u_{B_{2}}^{*}>u_{B}^{*}$

\section{Proposition 5.3}

Suppose $u_{S_{2}}^{*}<u_{S}^{*}$ and $u_{B_{2}}^{*}<u_{B}^{*}$ then $u_{C_{2}}^{*}>u_{C}^{*}$

Proof

Since $u_{S_{2}}^{*}<u_{S}^{*}$ and $u_{B_{2}}^{*}<u_{B}^{*}$ then,

$$
\begin{gathered}
u_{S_{2}}^{*}+u_{B_{2}}^{*}<u_{S}^{*}+u_{B}^{*} \\
1-\left(u_{S_{2}}^{*}+u_{B_{2}}^{*}\right)>1-\left(u_{S}^{*}+u_{B}^{*}\right) \\
1-u_{S_{2}}^{*}-u_{B_{2}}^{*}>1-u_{S}^{*}-u_{B}^{*}
\end{gathered}
$$

But $u_{C_{2}}^{*}=1-u_{S_{2}}^{*}-u_{B_{2}}^{*}$ and $u_{C}^{*}=1-u_{S}^{*}-u_{B}^{*}$, therefore

$$
u_{C_{2}}^{*}>u_{C}^{*}
$$

\section{Discussion and Conclusion}

\subsection{Discussion}

From proposition 5.1, we observed that with non stochastic salary, the optimal investment strategies for bond and stock increases with extra contribution while that of cash decreases with extra contribution. Also from proposition 5.2, we 
observed that with stochastic salary, the optimal investment strategies for bond and stock increases with extra contribution while that of cash decreases with extra contribution. We also had case where we could not conclude on the behavior of the optimal investment strategy for bond with extra contribution. In general we observed that extra contribution to the pension fund has an effect on the optimal investment strategies in cash, bond and stock. The analysis shows that the plan member will increase the proportion of his wealth to be invested in bond and stock and will reduce the proportion to be invested in cash.

\subsection{Conclusion}

The optimal investment strategy for a prospective investor in a DC pension scheme, with stochastic salary, under the affine interest rate model has been studied. Relevant to this work, the CRRA utility function was used and we obtained the optimal investment strategies for cash, bond and stock using the Legendre transform and dual theory. More so, the effect of various parameters were analyzed, in particular, the effect of extra defined contribution was $\mathrm{x}$-rayed, with significant input on the investment strategy.

\section{References}

[1] Cairns, A.J.G., Blake, D. and Dowd, K. (2006) Stochastic Lifestyling: Optimal Dynamic Asset Allocation for Defined Contribution Pension Plans. Journal of Economic Dynamics \& Control, 30, 843-877.

[2] Gao, J. (2008) Stochastic Optimal Control of DC Pension Funds. Insurance, 42, 1159-1164.

[3] Boulier, J.F. Huang, S. and Taillard, G. (2001) Optimal Management under Stochastic Interest Rates: The Case of a Protected Defined Contribution Pension Fund. Insurance, 28, 173-189.

[4] Deelstra, G., Grasselli, M. and Koehl, P.F. (2003) Optimal Investment Strategies in the Presence of a Minimum Guarantee. Insurance, 33, 189-207.

[5] Xiao, J., Hong, Z. and Qin, C. (2007) The Constant Elasticity of Variance (CEV) Model and the Legendre Transform-Dual Solution for Annuity Contracts. Insurance, $40,302-310$.

[6] Gao, J. (2009) Optimal Portfolios for DC Pension Plans under a CEV Model. Insurance, Mathematics and Economics, 44, 479-490. https://doi.org/10.1016/j.insmatheco.2009.01.005

[7] Chubing, Z. and Ximing, R. (2013) Optimal Investment Strategies for DC Pension with Stochastic Salary under Affine Interest Rate Model. Hindawi Publishing Corporation. https://doi.org/10.1155/2013/297875

[8] Othusitse Basimanebotlhe and Xiaoping Xue. (2015) Stochastic Optimal Investment under Inflamatory Market with Minimum Guarantee for DC Pension Plans. Journal of Mathematics, 7, 1-15.

[9] Cox, J.C., Ingersoll, J.E. and Ross, S.A. (1985) A Theory of the Term Structure of Interest Rates. Econometrica, 53, 385-407. https://doi.org/10.2307/1911242

[10] Vasicek, O. (1977) An Equilibrium Characterization of the Term Structure. Journal of Financial Economics, 5, 177-188. https://doi.org/10.1016/0304-405X(77)90016-2

[11] Duffie, D. and Kan, R. (1996) A Yield-Factor Model of Interest Rates. "Mathemati- 
cal Finance, 6, 379-406. https://doi.org/10.1111/j.1467-9965.1996.tb00123.x

[12] Battocchio, P. and Menoncin, F. (2004) Optimal Pension Management in a Stochastic Framework. Insurance, 34, 79-95.

[13] Jonsson, M. and Sircar, R. (2002) Optimal Investment Problems and Volatility Homogenization Approximations. Modern Methods in Scientific Computing and Applications, 75, 255-281. 